Article

\title{
Numerical Study of the Air Flow over a NACA 0015 Wind Turbine Airfoil
}

\author{
Houari Ameur *, Karima Boukhadia \\ Institute of Science and Technology, University Center Salhi Ahmed of Naâma (Ctr Univ Naâma), P.B. 66, \\ 45000, Algeria \\ * Corresponding author, E-mail address: houari_ameur@yahoo.fr; h.ameur@mail.cuniv-naama.dz; Tel: \\ $+213770343722$
}

\begin{abstract}
A numerical study of the flow over a NACA aerofoil is presented in this paper. The numerical simulations are achieved with the computer code CFX and the computational domain is created by the computer tool ANSYS ICEM CFD. The CFX code is based on the finite volume method to solve the equations of mass, momentum and energy. The purpose of this paper is to determine the pressure distribution, flow patterns and the forces acting on the airfoil. Effects of the attack angle and Reynolds number on the velocity and pressure distribution, on the lift and drag coefficients are also explored.
\end{abstract}

Keywords: CFD; NACA airfoil; Wind turbine; Attack angle; Wall pressure.

\section{Introduction}

Up today, the energy demand knows an exponential increase in the daily human life. However, the resources of energy fossil are very limited and the environmental pollution is becoming a serious problem. Therefore, the renewable energy such as wind turbine is considered as an emergency alternative resource.

The performance of wind turbines in terms of transformation of wind energy to electrical energy is jugged as not good; so many studies have been achieved on the design parameters of wind turbines, the so-called airfoil profile [1-3].

Troolin et al. [4] explored the efficiency of a new design: Gurney flap added with NACA 0015 airfoil. Their results showed no change in the drag coefficient and just an increase in the lift coefficient. Siauw et al. [5] studied the transient dynamics of the flow around the NACA 0015 airfoil by using fluid vortex generator. Şahin and Acir [6] studied the turbulent flow over a NACA 0015 for different turbulence models.

Ismail and Vijayaraghavan [7] explored the effect of profile-modifications on a NACA-0015 aerofoil employed in vertical axis wind turbines. The modification added is a combination of inward semi-circular dimple and Gurney flap at the lower surface of the NACA-0015 aerofoil. By detached-eddy simulation, Wang and Fu [8] studied the flow over a NACA 0015 airfoil with pulsed actuation at an incidence angle of $11^{\circ}$. Siauw and Bonnet [9] studied the transient phenomena occurring during the impulsive control of flow separation over a NACA0015 airfoil at an incidence angle of $11^{\circ}$ and a chord Reynolds number of 1 million.

The present paper is a numerical study of the subsonic and two-dimensional flow over a NACA 0015. We explore here the effect of Reynolds number and attack angle. 


\section{Presentation of the Problem}

The airfoil simulated is a NACA 0015. For this type of airfoils, the maximal width is located at $15 \%$ of the length of airfoil from the leading edge (Figure 1). The working fluid is air and the study is two-dimensional. The boundary conditions are provided in Figure 1.

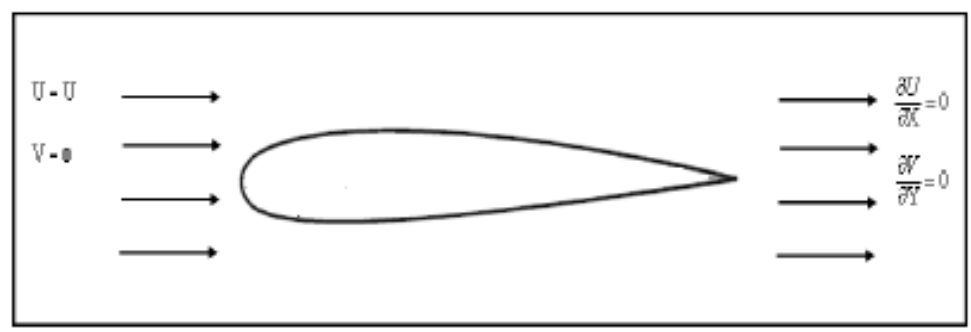

Figure 1. NACA 0015 airfoil (boundary conditions)

\section{Numerical and Theoretical Details}

For the air flow field, the steady-state, Newtonian and incompressible Reynolds-averaged Navier-Stokes equations of mass, momentum and energy are solved. The $k-\varepsilon$ turbulence model is used to model the turbulence effect. The pressure-based segregated SIMPLE (Semi-Implicit Method for Pressure Linked Equations) algorithm is used for pressure-correlation equation calculation. Cao et al. [10] have adopted this algorithm for the simulation of parachute aerodynamics. For spatial discretization, the pressure term uses second order scheme, the momentum, energy and turbulence terms use the second-order accurate QUICK scheme. We note that the QUICK scheme is based on a weighted average of the second-order central and upwind interpolations of the discretized variable. Further detail may be found elsewhere [11].

We define the dimensionless longitudinal and vertical coordinates as: $X^{*}=X / C, Y^{*}=Y / C$, respectively. The dimensionless velocity is given by $V^{*}=V / V_{\text {in }}$, where $V_{\text {in }}$ is the inlet velocity at the computational domain. Reynolds number $(R e)$ is defined as:

$$
\operatorname{Re}=\frac{V_{\text {in }} C}{\mu}
$$

Where $C$ is the airfoil cord length.

Airfoils have different sizes and shape. Therefore and in order to determine the performance (the advantages and disadvantages) of these airfoils, non-dimensional coefficients (lift and drag coefficients) were taken into consideration. The non-dimensional coefficients for two dimensions solution were given as below:

Lift coefficient:

$$
C_{\mathrm{L}}=\frac{2 L}{\rho V_{i n}^{2} C}
$$

$$
\text { Drag coefficient: }
$$

$$
C_{\mathrm{D}}=\frac{2 D}{\rho V_{i n}^{2} C}
$$

where $L$ and $D$ are lift and drag force respectively, $C_{\mathrm{L}}$ and $C_{\mathrm{D}}$ are lift and drag coefficient of airfoil respectively, $V_{\text {in }}$ is velocity of wind, $\rho$ is density of air.

\section{Results and Discussion}

\subsection{Pressure and velocity fields}

The distribution of pressure and velocity fields are provided on Figure 2 for a Reynolds number $R e=1.48 \times 10^{3}$. For an attack angle $\alpha=0^{\circ}$, the pressure fields are the same on both surfaces of the airfoil. The high value of pressure acting on the wall airfoil is located at the attack edge. Then, negative values of pressure gradients are observed along the surfaces of the airfoil, which mean the 
80

81

82

83

presence of detached boundary layer. The flow patterns are also identical on both sides of the NACA airfoil, where the maximum velocity is observed near the leading edge. However, the minimum is located near the trailing edge where trailing vortices may be generated for inclined NACA airfoils.

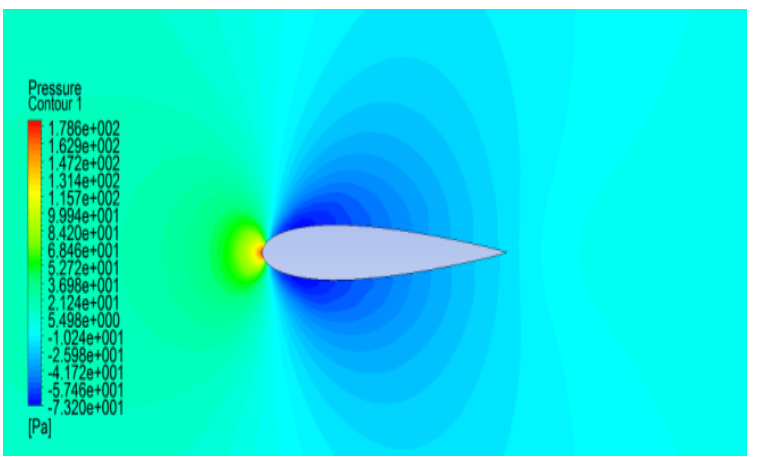

(a) Pressure field

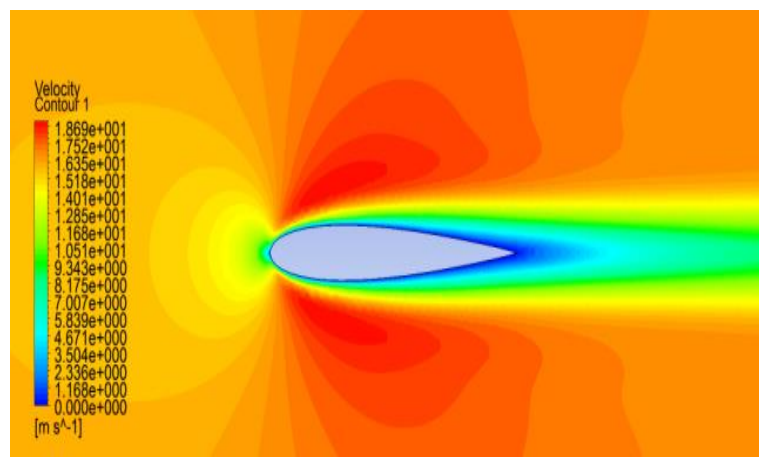

(b) Velocity field

Figure 2. Distribution of (a) pressure and (b) velocity on the NACA airfoil, at $\alpha=0^{\circ}, R e=1.48 \times 10^{3}$

\subsection{Effect of Reynolds number}

Three values of Re are chosen to explore the effect of Reynolds number, namely $R e=1.48 \times 10^{3}$, $1.86 \times 10^{3}$ and $2.79 \times 10^{3}$. The velocity field is presented on Figure 3 for different Re. As observed, the increase of $R e$ yields an intensified movement of fluid particles, giving thus a reduction in the thickness of boundary layer. This finding is in agreement with theoretical background: the width of the boundary layer is defined as: $\delta=(0.37 \mathrm{X}) /(\operatorname{Re} 0.2)$.

Figure 4 shows the variation of axial velocity along the vertical position at $X / C=0.9$ (i.e. near the trailing edge). The velocity increases rapidly along the vertical position until the height $X / C=0.2$ where this parameter remains almost constant.

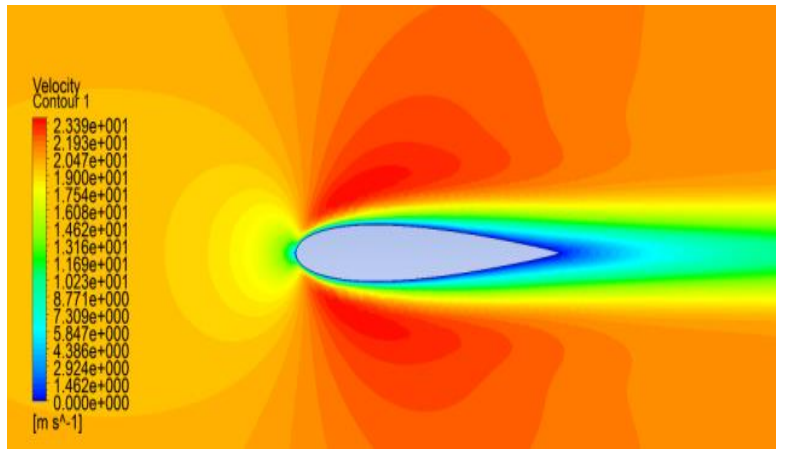

$R e=1.86 \times 10^{3}$

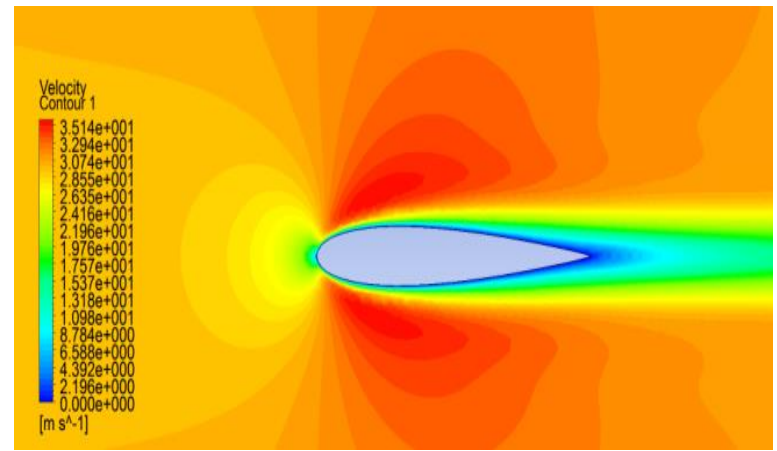

$\operatorname{Re}=2.79 \times 10^{3}$

Figure 3. Velocity distribution for different Reynolds numbers, $\alpha=0^{\circ}$

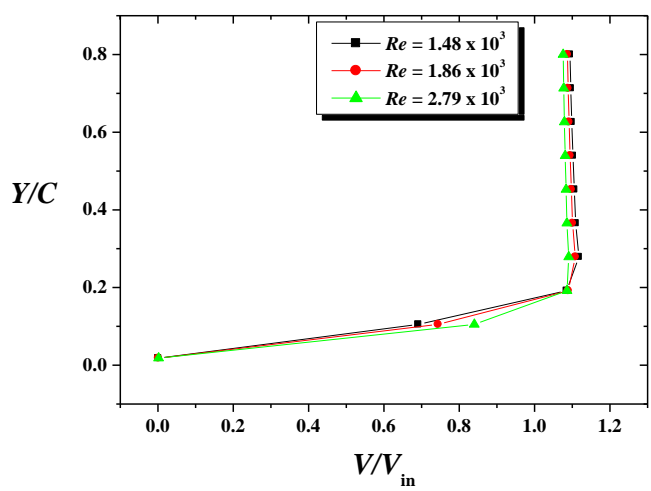

Figure 4. Dimensionless velocity for different Reynolds numbers, at $X / C=0.9, \alpha=0^{\circ}$ 


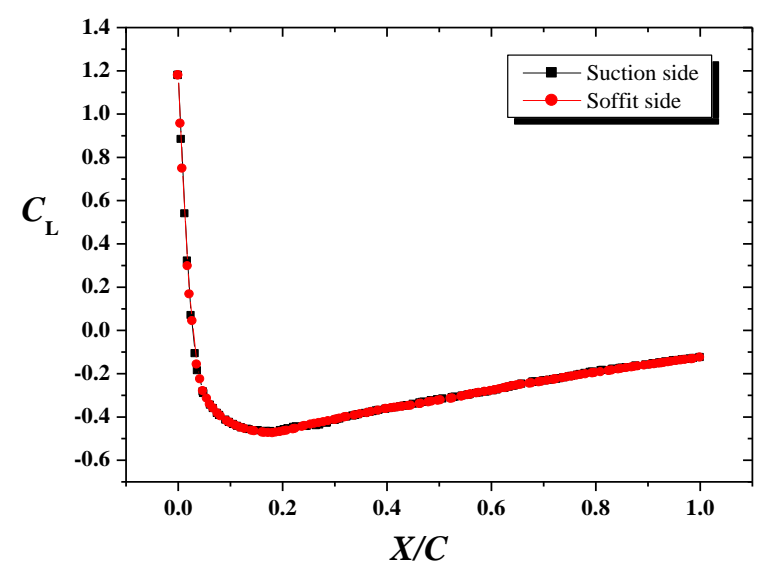

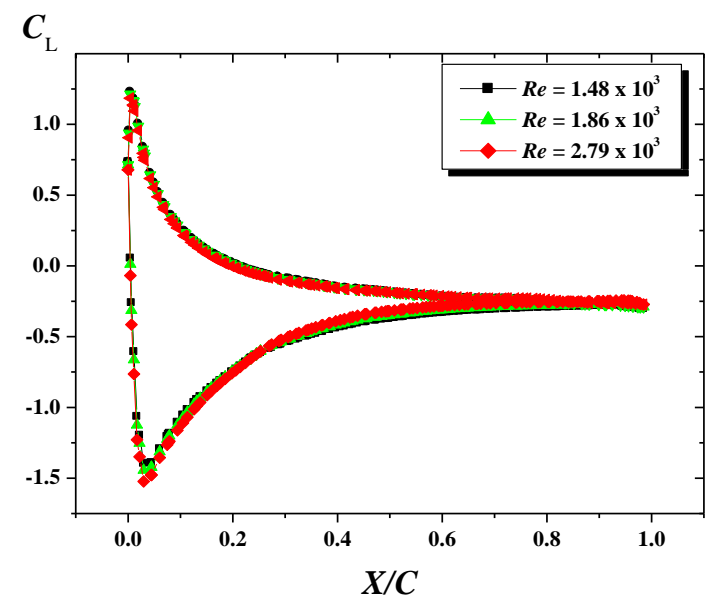

Figure 6. Variation of $C_{\mathrm{L}}$ for different Reynolds numbers, $\alpha=0^{\circ}$ Reynolds number for the range studied here (Figure 6).

Figure 5. Distribution of the lift coefficient on the surfaces of NACA 0015 airfoil, at $\alpha=0^{\circ}$

Variations of lift coefficient are plotted on Figure 5 for both sides of the NACA airfoil. At the attack angle $\alpha=0^{\circ}$ and for a symmetric airfoil, the lift coefficient follows the same profile on both sides of the NACA. The maximum value is observed at the leading edge, followed by a strong decrease until the axial position $X / C=0.15$ (i.e. at the maximum width of the airfoil), then $C_{\mathrm{L}}$ increases newly until the trailing edge. However, the lift coefficient is found to be independent of

Figure 7 presents the variation of drag coefficient for different Reynolds numbers. It is clear that

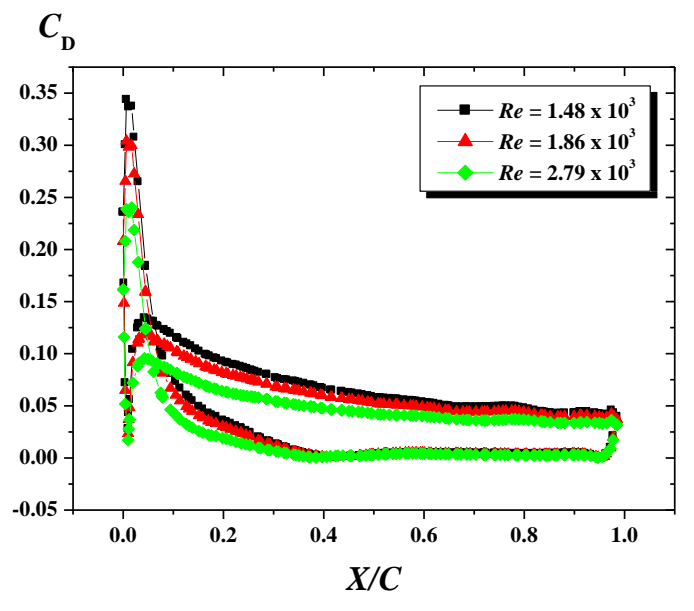

Figure 7. Variation of $C_{D}$ for different Reynolds numbers, $\alpha=10^{\circ}$ $C_{D}$ reaches its maximum value at the leading edge (at the point of stagnation) and it decreases continually along the airfoil surface. Also, the drag coefficient decreases with the rise of Reynolds number.

\subsection{Effect of attack angle}

In this section of paper, we investigate the effect of attack angle $(\alpha)$ on the lift and drag forces. Therefore, four cases are explored, which are: $\alpha=0^{\circ}, 8^{\circ}, 10^{\circ}$ and $15^{\circ}$. Figures 8 and 9 present the variation of lift coefficient and drag coefficient along the length of airfoil for different attack angles, respectively. As shown on these Figures, the increase of attack angle until $15^{\circ}$ yields an increase in lift and drag coefficients. 


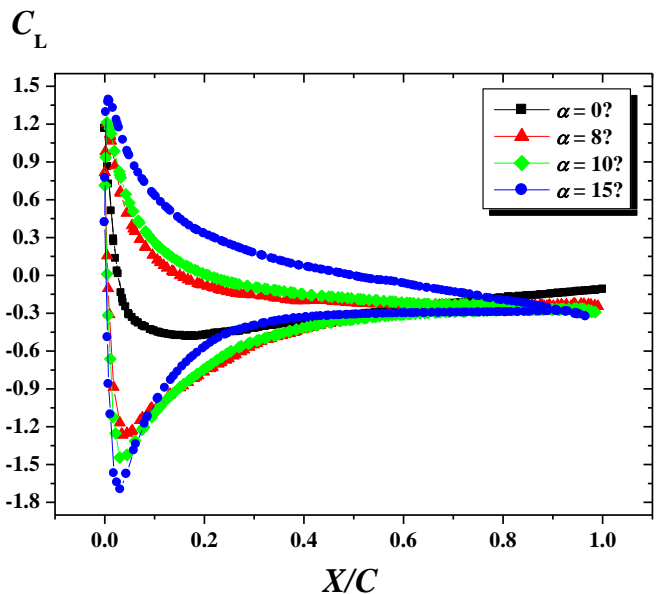

Figure 8. Distribution of $C_{\mathrm{L}}$ for different attack angles, at $R e=1.86 \times 10^{3}$

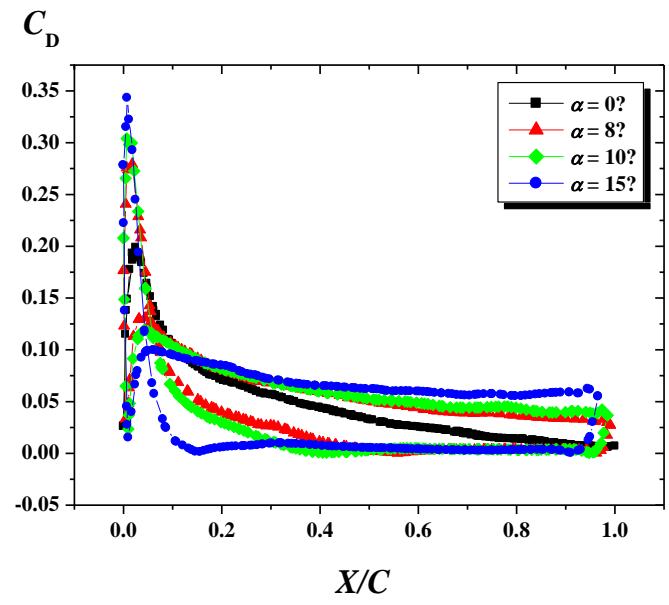

Figure 9. Distribution of $C_{D}$ for different attack angles, at $R e=1.86 \times 10^{3}$

\section{6}

117

118

119

120

121

122

123

124

125

126

127

128

129

130

131

132

133

134

135

136

137

138

139

140

141

142

143

\section{Conclusion}

In this paper, lift and drag performances of NACA 0015 airfoil were performed. A CFX program was employed to achieve numerical calculations. The pressure field and flow patterns are found in good agreement as theoretical findings. The predicted results were given as follows:

- The velocity is neglected at the leading edge, and then it increases continually until reaching the magnitude of the uniform flow. These velocity gradients determine the height of boundary layer.

- $\quad$ Drag and lift coefficients increased with increasing attack angle.

- The Reynolds number has no effect on the distribution of static pressure (i.e. on the lift coefficient). However, the drag coefficient decreases with the rise of $R e$.

\section{References}

1. Wu, Z.; Cao, Y. Numerical simulation of flow over an airfoil in heavy rain via a two-way coupled Eulerian-Lagrangian approach. Int. J. Multi. Flow 2015, 69, 81-92.

2. Sato, M.; Asada, K.; Nonomura, T.; Kawai, S.; Fujii, K. Large-eddy simulation of NACA 0015 airfoil flow at Reynolds number of $1.6 \times 10^{6}$. AIAA J. 2017, 55, 673-679.

3. Nekoubin, N.; Nobari, M.R.H. Numerical investigation of transonic flow over deformable airfoil with plunging motion. Appl. Math. Mech. Eng. Edit. 2016, 37, 75-96.

4. Troolin, D.R.; Longmire, E.K.; Lai, W.T. Time resolved PIV analysis of flow over a NACA 0015 airfoil with Gurney flap. Exp. Fluid 2006, 41, 241-254.

5. Siauw, W.L.; Bonnet, J.-P.; Tensi, J.; Cordier, L.; Noack, B.R.; Cattafesta, L. Transient Dynamics of the flow around a NACA 0015 airfoil using fluidic vortex generators. Int. J. Heat Fluid Flow 2010, 31, 450-459.

6. Şahin, İ.; Acir, A. Numerical and experimental investigations of lift and drag performances of NACA 0015 wind turbine airfoil. Int. J. Mat. Mech. Man. 2015, 3, 22-25.

7. Ismail, M. F.; Vijayaraghavan, K. The effects of aerofoil profile modification on a vertical axis wind turbine performance. Energy 2015, 80, 20-31.

8. Wang, L.; Fu, S. Detached-eddy simulation of flow past a pitching NACA 0015 airfoil with pulsed actuation. Aerospace Sci. Technol. 2017, 69, 123-135.

9. Siauw, W.L.; Bonnet, J.P. Transient phenomena in separation control over a NACA 0015 airfoil. Int. J. Heat Fluid Flow 2017, 67, 23-29. 
145 10. Cao, Y.H.; Wang, K.; Song, Q.F. Numerical simulation of parachute fluid-structure interaction in terminal 146 descent. Sci. China Technol. Sci. 2012, 55, 3131-3141.

147 11. Wu, Z.; Cao, Y. Numerical simulation of flow over an airfoil in heavy rain via a two-way coupled 148 Eulerian-Lagrangian approach. Int. J. Multi. Flow 2015, 69, 81-92. 